\title{
Long non-coding RNA RMRP promotes human glioma tumor progression via miR-466/CXCR4 pathway
}

Jie Zhang ( $\nabla$ sij5y7@163.com )

The First Affiliated Hospital of Chengdu Medical College https://orcid.org/0000-0002-7937-6750 Ling He

The First Affiliated Hospital of Chengdu Medical College

\section{Xun Xia}

The First Affiliated Hospital of Chengdu Medical College

\section{Lie Zhang}

The First Affiliated Hospital of Chengdu Medical College

\section{Enren Wang}

The First Affiliated Hospital of Chengdu Medical College

\section{Research}

Keywords: IncRNA RMRP, miR-466, CXCR4, tumor progression, glioma

Posted Date: March 17th, 2021

DOI: https://doi.org/10.21203/rs.3.rs-315866/v1

License: (c) (1) This work is licensed under a Creative Commons Attribution 4.0 International License. Read Full License 


\section{Abstract}

Purpose: Long non-coding RNA (IncRNA) RMRP has been confirmed to be associated with multiple tumor progression. However, the molecular regulatory mechanism of RMRP in human glioma was rarely elucidated.

Methods: The quantitative real-time polymerase chain reaction (qRT-PCR) was used to measure the expression levels of RMRP, microRNA-466 (miR-466) and CXCR4 in glioma tissues and cells. The ELISABrdU assay and flow cytometry analysis was used to perform the cell proliferation, apoptotic rate, cell cycle assay in human glioma cell lines A172 and U251 cells. The starBase v2.0 or TargetScan was used to predict the potential interactions among RMRP, miR-466 and CXCR4, and the luciferase reporter assay was performed to validate the relationship among these molecules. The western blot was used to determine the expression levels of protein associated with cell cycle and CXCR4.

Results: The levels of RMRP, CXCR4 were highly expressed, but miR-466 was decreased in glioma tissues and cells. And miR-466 was verified as a direct target of RMRP and directly regulated CXCR4. RMRP expression or miR-466 inhibitor promote cell proliferation and G1/S cell cycle transition but reduced apoptotic rate in A172 and U251 cells, while RMRP promote G1/S cell cycle transition through regulating CDK4 pathway, not CDK2 pathway. And that, the introduction of CXCR4 partly counteracted these impacts. In addition, RMRP silencing and miR-466 mimic impeded xenograft tumor growth in vivo.

Conclusion: RMRP enhanced glioma progression, partially through miR-466/CXCR4 pathway. The molecular regulation mechanism based on RMRP/miR-466/CXCR4 might contribute to the diagnosis of glioma and provide the effective therapeutic targets for its treatment.

\section{Introduction}

Glioma is the most common tumor of the nervous system [1]. In addition, gliomas are typically characterized by malignant hyperplasia and aggressive growth with a high recurrence rate and poor prognosis. Therefore, it is difficult to cure glioma completely with traditional treatment methods. Hence, Therefore, it is urgent to explore the mechanism of glioma development and identify new therapeutic targets.

Non-coding RNAs (ncRNAs) are known to influence tumor progression through a variety of regulatory pathways. [2]. Long non-coding RNAs (IncRNAs, $>200 \mathrm{nts}$ ) are non-translational ncRNAs that regulate gene expression in tumorigenesis and progression [3]. Previous studies have identified multiple IncRNAs up-regulated in gliomas. For instance, IncRNA H19 was increased in glioma and promoted glioma angiogenesis via binding miR-138 and regulating HIF-1a/VEGF axis [4]. Another study demonstrated that IncRNA PVT1 facilitated tumorigenesis and progression in human glioma, and confirmed a regulatory relationship between PVT1 and miR-128-3p as well as that between miR-128-3p and GREM1 [5]. The RNA component of mitochondrial RNA processing endoribonuclease (RMRP) is a $277 \mathrm{nt}$-long IncRNA molecule, and increasing evidence has demonstrated that it is a novel oncogene, the expression level of 
which is increased in bladder cancer and non-small cell lung cancer [6, 7]. For example, Zhao et al. reported that IncRNA RMRP knockdown could suppress hepatocellular carcinoma and targeted miR-206 to regulat TACR1 to inhibit cell proliferation [8]. However, IncRNAs often target multiple miRNAs and thus have multiple regulatory functions. Therefore, it is necessary to further elucidate the regulatory mechanisms of the oncogene RMRP in the progression of human glioma.

MicroRNAs (miRNAs) are another type of non-coding RNAs that primarily perform RNA silencing functions [9]. The miRNA is involved in almost all the developmental and pathological processes in animals, and its disturbance is associated with many human diseases, including tumor progression [10]. LncRNAs can inhibit miRNA expression through competitive binding of miRNAs, and thus exert different biological functions in negative regulation of downstream target genes [11]. Overexpression of IncRNA or interrupting the expression of miRNAs can promote the proliferation, invasion, migration, and apoptosis of tumors. Some previous researches have indicated that miR-466 was significantly downregulated, which might be potential cancer suppressors in different types of cancers, such as neuroblastoma colorectal cancer, prostate carcinoma, and hepatocellular carcinoma [12-14]. Nevertheless, the regulatory function of IncRNA RMRP/miR-466 axis in glioma cells needs to be further elucidated.

Herein, our study aims to investigate the explicit role of IncRNA RMRP in the pathogenesis of glioma as well as the potential regulatory mechanism between IncRNA RMRP and miR-466 in human glioma progression. We delved into the IncRNA-miRNA-mRNA networks in occurrence and development of human glioma and provide a potential novel biomarker or therapeutic target for the prognosis and treat of human glioma.

\section{Materials And Methods}

\section{Patients and Clinical tissue samples}

Fifty-eight patients who underwent surgery at the Department of Neurosurgery in the First Affiliated of Chengdu Medical College from July 2015 to September 2016 were included in the study, and classified according to the WHO guidelines for the classification of tumours of the central nervous system [15]. This study mainly collected the information from the patients' medical records, and the detail information was showed in Table 1. The survival rate was calculated from the data of surgical resection to Dec 31, 2020. The human glioma tissue of the patients or the control group was immediately immersed in liquid nitrogen until RNA extraction.

The written informed consents were collected from all patients, and this study was approved by the Ethics Committee of the First Affiliated of Chengdu Medical College (IRB number: 20140332). 
Table 1

Clinicopathological characterisitcs of RMRP expression in glioma patients.

Clinical feature

Age

$<60$

$\geq 60$

Gender

Male

Female

Histologic Type

Astrocytoma

Glioblastoma

Oligoastrocytoma

Oligodendroglioma

WHO Grade

Grade II

Grade III

Grade IV

Recurrence Status

No

Yes

Recurrence time in months [mean (sd)]

Survival Status

Alive
RMRP expression"

High

Low

18

10

17

13

7 $p$ value

$0.076 *$
0.368

$14 \quad 16$

$14 \quad 14$

$0.041 *$

2

20

16

1

5

3

7

4

$0.024 *$

5

10

6

13

19

5

0.434

$19 \quad 20$

$9 \quad 10$

$23.4(25.3)$

14.5 (16.4)

0.121

0.009 *

20

9

${ }^{*} p<0.05$ was considered statistically significant.

\# Low/high expression was determined by the sample mean. Pearson chi-square test was utilized to analyze the clinical data.

${ }^{\&} p$-value from $t$ test. 


\begin{tabular}{|c|c|c|c|}
\hline \multirow[t]{2}{*}{ Clinical feature } & \multicolumn{2}{|c|}{ RMRP expression\# } & \multirow[t]{2}{*}{$p$ value } \\
\hline & Low & High & \\
\hline Deceased & 8 & 21 & \\
\hline Survival time in months [mean (sd)] $]^{\&}$ & $52(32.2)$ & $21.3(23.5)$ & $0.003^{*}$ \\
\hline \multicolumn{3}{|l|}{ IDH1 mutation } & \multirow[t]{3}{*}{$0.037 *$} \\
\hline Negative & 15 & 19 & \\
\hline Positive & 13 & 11 & \\
\hline \multicolumn{4}{|c|}{${ }^{*} p<0.05$ was considered statistically significant. } \\
\hline \multicolumn{4}{|c|}{$\begin{array}{l}\text { \# Low/high expression was determined by the sample mean. Pearson chi-square test was utilized to } \\
\text { analyze the clinical data. }\end{array}$} \\
\hline \multicolumn{4}{|l|}{$\&_{p \text {-value from } t \text { test. }}$} \\
\hline
\end{tabular}

\section{Cell culture}

The human glioma cell lines A172, SHG44, U251, LN229 as well as the normal human astrocytes (NHAs) were obtained from the American Type Culture Collection (Manassas, VA, USA). All cell lines were maintained in DMEM medium (Gibco, Thermo Fisher Scientific, Waltham, MA, USA) containing 10\% FBS (Gibco) and cultured in a $5 \% \mathrm{CO}_{2}$ incubator at $37^{\circ} \mathrm{C}$.

\section{RNA extraction and quantitative RT-PCR analysis}

The total RNA was isolated from human glioma tissues and cell lines using TRIzol reagent (Invitrogen).

For quantitative RT-PCR, the total RNA was reverse-transcribed into complementary DNA using the reverse transcription kit (Invitrogen), according to the manufacturer's instruction. Further, the SYBR green PCR kit (Toyobo, Nipro, Osaka, Japan) was used to conduct real-time PCR with the 7500 Fast Real-time PCR system (Applied Biosystems, Waltham, MA, USA). The $2^{-\triangle \Delta C t}$ method was used for calculating the target gene expression levels, and GAPDH or U6, as endogenous control, was used to normalize the target gene expression levels, respectively. The primer sequences were as follows: RMRP, F-5'CACTGCCTATGTGCACGACT-3', R-5'AGAGTCCGGCAAGAAGAACA-3'; miR-466, F-5'GGGATTCCCCTGGGTGTCAACACTGGC-3', R-5'-TGCGCAGCACAGTTCCACGGGTC-3'; CXCR4, F-5'ACCCCTTCATTGACCTCAACTA-3', R-5'-TCTCGCTCCTGGAAGATGGTGA-3'; GAPDH: F-5'AATGGGCAGCCGTTAGGAAA-3', R-5'TGAAGGGGTCATTGATGGCA-3'; and U6: F-5'CTCGCTTCGGCAGCACATATA-3', R-5'ACGCTTCACGAATTTGAGTGTC-3'. All assays were performed three times.

\section{Cell transfection}


The small short hairpin RNA (shRNA) targeting RMRP, control shRNA, pcDNA-RMRP, and the control plasmid pcDNA3.1 were provided by Novogene (Beijing, China). The detailed sequences of all targeted genes were used: shRMRP: (F) 5'GGCCAAACCCUCAAUGAAUTT-3' and (R) 5'AUUCAUUGAGGGUUUGGCCTG-3'; shCtrl: (F) 5'UUCUCCGAACGUGUCACGUTT-3' and (R) 5'ACGUGACACGUUCGGAGAATT-3'; pcDNA-RMRP: (F) 5'CGATCTTAATTAAGGGGTACCAAAGTCCACTCTG3 ' and (R) 5'TCAGTGGCGCGCCTTTTTCGTGAGTACACAATAGTCATC-3'. The miR-466 mimic (5'ACCUGGCAUACAAUGUAGAUUU-3'), miR-negative control (NC) mimic (5'-UUCUCCGAACGUGUCACGUTT3'), miR-466 inhibitor (5'-UGAGGUAGUAGGUUGUAUAGUU-3) and miR-NC inhibitor (5'-

CAGUACUUUUGUGUAGUACAA-3') were provided by Shanghai Sangon Biotech Corporation. The corresponding plasmid or miRNAs ( $50 \mathrm{nM}$ ) were transfected into A172 and U251 cell lines with a density of $10^{5}$ /well in 6-well plates by Lipofectamine 2000 reagent (GE Healthcare Life Sciences, Lafayette, CO, USA), and the cells were collected for analysis at $48 \mathrm{~h}$ after transfection.

\section{MTT assays}

A172 and U251 cell lines were added to a 96-well dish at a density of $10^{3}$ cells per well and then transfected with pcDNA-RMRP, sh-RMRP, miRNA, pcDNA-CXCR4, or shCXCR4. Subsequently, at 0, 24, 48, 72 , and $96 \mathrm{~h}, 10 \mu \mathrm{lMTT}$ solution was added to each well. After $4 \mathrm{~h}$ of culture, the supernatant was discarded, and then $100 \mu \mathrm{l}$ of DMSO was added. The absorbance at $570 \mathrm{~nm}$ was determined using an enzyme-labeling instrument (EL808 BioTek Instruments, Winooski, VT, USA).

\section{BrdU proliferation assay}

A cell proliferation assay was performed using a cell proliferation ELISA-BrdU (colorimetric) Kit (Roche Diagnostics, CA, USA), according to the manufacturer's protocol. Briefly, the A172 and U251 cell lines were cultured into a 96-well plate at a density of $10^{3}$ cells per well. Overnight, the cells were transfected with the corresponding pcDNA-RMRP, sh-RMRP, miRNA, pcDNA-CXCR4, or shCXCR4 at $37^{\circ} \mathrm{C}$. After $24 \mathrm{~h}$ of incubation, cell proliferation was analyzed.

\section{Apoptosis analysis}

The A172 and U251 cells were seeded into 6-well plates at a density of $10^{5}$ cells per well, and the pcDNARMRP, sh-RMRP, miRNA, pcDNA-CXCR4, or shCXCR4 were transfected into cells. After that, a cell apoptosis assay was conducted via flow cytometry using the Annexin V-FITC Apoptosis Detection Kit (Beyotime, China). Briefly, cells were collected with annexin-V/FITC $(5 \mu \mathrm{l})$ and propidium iodide $(10 \mu \mathrm{l})$, and were incubated for $15 \mathrm{~min}$ at room temperature in the dark. The cell apoptosis rate was analyzed within $1 \mathrm{~h}$ using a FACS caliber cytometer (BD Biosciences, Franklin Lakes, NJ, USA).

\section{Cell cycle analysis}

The A172 and U251 cells were transfected with pcDNA-RMRP, sh-RMRP, miRNA, pcDNA-CXCR4, or shCXCR4 for $24 \mathrm{~h}$. After that, the cells were collected by trypsinization and washed with PBS that was precooled on ice. The cells were fixed in $70 \%$ methanol and incubated at $4{ }^{\circ} \mathrm{C}$ for $1 \mathrm{~h}$. The cells were then centrifuged and added with RNase at $37^{\circ} \mathrm{C}$ and incubated for $30 \mathrm{~min}$. The cells were stained by 
propidium iodide for $1 \mathrm{~h}$. The cell cycle of pretreated cells was analyzed using FACScan flow cytometer, and the data were presented by CellQuest software.

\section{Western blotting}

The Western blot assay used in this study was according to the following previously described methods. Total proteins were extracted from A172 cells using a protein isolation kit (Tiangen, Beijing, China), and the protein concentrations were quantified through the Pierce BCA assay (Thermo Scientific, CA, USA). Equal amounts of protein samples were separated by SDS-PAGE and then electrophoretically transferred to a polyvinylidene fluoride membrane (Milipore, CA, USA). The antibodies against CDK4, Cyclin-D1, CDK2, Cyclin-E, $\beta$-actin, and CXCR4 (Santa Cruz Biotechnology, CA, USA) as the primary antibody (all obtained from Cell signaling Technology, Beverly, MA, USA) were used in the Western blot assay, and peroxidase-conjugated anti-IgG (Abcam) was also used as the secondary antibody. Monoclonal $\beta$-actin or GAPDH antibody (Sigma) was used as the control. The blots were detected using an enhanced chemiluminescent kit (Pierce, Rockford, IL, USA). The data of Western blot bands were quantified by Image $\mathrm{J}$ software.

\section{Tumorigenesis analysis}

The nude mice were used to determine the tumorigenesis as described previously. Briefly, 4-week-old female BALB/c nude mice were subcutaneously injected with U373MG cells ( $10^{5}$ cell in $200 \mathrm{~mL} / \mathrm{mouse}$ ) transfected with shRMRP, shCtrl, miR-466 mimic or mimic control at a single site under their right front leg. After 2 weeks, the tumor sizes were measured every 3 days. After 5 tests (14, 17, 20, 23and 26 days), the tumor volumes were calculated. The average weight of the excised tumor was measured at the time of sacrifice at 5 weeks after the initial injection.

All the procedures were approved by the Animal Management rule of the First Affiliated of Chengdu Medical College.

\section{Statistical analysis}

All experiments were carried out in triplicate, and the GraphPad Prism 5 software (GraphPad Software Inc., San Diego, CA, USA) was used for all statistical analyses. Data for each group of each experiment were presented as mean \pm standard deviation (SD). The overall survival rate was calculated using the Kaplan-Meier method. Two-tailed Student's t-test or analysis of variance followed by Turkey's post hoc test was applied to analyze significance, and the differences were considered statistically significant at $P$ $<0.01$.

\section{Results}

\section{The expression level of IncRNA RMRP is upregulated in human glioma}


To investigate the correlation between RMRP and human glioma, RT-qPCR was used to detect the levels of RMRP in 58 pairs of human glioma tissue samples and corresponding non-tumor tissue samples. As shown in Fig. 1A, the expression of RMRP was clearly higher in human glioma tissues relative to nontumor tissues (Fig. 1A). The data of overall survival rate also showed that human glioma patients with high RMRP level had worse survival than those with low RMRP level (Fig. 1B). In addition, the clinical data confirmed that the expression level of RMRP was significantly related to WHO grades, histological type but not with patient's age and gender (Table 1). Further, the increased expression levels of RMRP were also confirmed in human glioma cell lines A172, SHG44, U251, LN229 and the normal human astrocytes (NHAs) (Fig. 1C). Taken together, our findings indicate that RMRP expression is upregulated in human glioma, and closely related to the development of this cancer.

RMRP increases cell proliferation, reduces apoptosis of glioma cells and promotes cell cycle via regulating CDK4/Cyclin-D1 expression

To determine the relationship between RMRP and human glioma progression, the pcDNA-RMRP and shRMRP were transfected into the human glioma cell lines A172 and U251 (the levels of RMRP in these cell lines was higher than other cell lines in previous experiment) to cause overexpression or silencing of RMRP, respectively. In addition, the comparative analysis result is that the control data of pcDNA3.1 and shCtrl are taken as negative control. Analysis showed that, compared to the negative control, RMRP promoted cell viability and proliferation (Fig. 2A and B). In contrast, RMRP decreased the apoptosis of human glioma cells (Fig. 2C). Collectively, these data suggest that RMRP promotes cell proliferation and attenuates apoptosis in human glioma.

Because the RMRP effectively promoted human glioma cell proliferation, we then evaluated the effect of RMRP on the cell cycle of human glioma cells and confirmed this using flow cytometry. As shown in Fig. 2D, compared with cells transfected with shRMRP, pcDNA-RMRP significantly reduced the percentage of $\mathrm{G} 1 / \mathrm{G} 0$ peak cells and increased the percentage of $S$ peak cells, which suggested that RMRP may promote the cell proliferation by accelerating the G1/S cell cycle transition. And then, we investigated the mechanisms by which RMRP regulates the cell cycle. As we know, G1/S cell cycle transition is governed by cyclin D-CDK4/6 and cyclin E-CDK2 complexes $[16,17]$. Therefore, we examined the effects of the RMRP on cyclin D-CDK4/6 and cyclin E-CDK2 expression. As shown in Fig. 2E and F, CDK4 and Cyclin-D1 protein expressions were upregulated in human glioma cells transfected with the pcDNA-RMRP compared with those cells transfected with shRMRP. Besides, CDK2 and Cyclin-E protein expression levels did not change much in pcDNA-RMRP-transfected cells compared with those in the control. These data revealed that RMRP could accelerate cell cycle by regulating cyclin D-CDK4 complex expression.

\section{miR-466 is a direct target of RMRP}

Further, we found that miR-466 was predicted as a direct target of RMRP via the DIANA tool (Fig. 3A). And that, the expression levels of miR-466 was decreased in various human glioma cell lines (Fig. 3B). The data of luciferase reporter assay showed that miR-466 mimic could direct interact with RMRP (Fig. 3C). Moreover, the expression levels of miR-466 in cells transfected with the pcDNA-RMRP or shRMRP was 
examined by RT-qPCR. The data showed that miR-466 levels was obviously decreased by the pcDNARMRP but increased by the shRMRP (Fig. 3D). And that, the expression levels of RMRP also affect by the miR-466 mimic or miR-466 inhibitor (Fig. 3E).

To confirm the biological function of miR-466 targeting RMRP in the progression of human glioma, miR466 mimics and pcDNA-RMRP were co-transfected into glioma cells and cell proliferation assay and flow cytometry were performed. As shown in Fig. 3F and G, miR-466 mimics reversed the reduction of cell viability and proliferation induced by overexpressing RMRP. Subsequently, miR-466 rescued the apoptosis induced by the overexpression of RMRP (Fig. 3H). Similarly, miR-23b mimics also largely reversed the block effects of RMRP on human glioma cell cycle (Fig. 3I). Taken together, these results further confirmed that RMRP could promote human glioma progression via targeting miR-466.

\section{CXCR4 bound to miR-466, and promoted cell proliferation but reduced apoptosis to human glioma cells}

Similar with the RMRP target to miR-466, miR-466 could target to CXCR4 via the DIANA tool (Fig. 4A). And that, the expression levels of CXCR4 was increased in various human glioma cell lines (Fig. 4B). The dualluciferase reporter gene assay also confirmed this interaction between miR-466 and CXCR4 (Fig. 4C). After verifying the transfection efficacy of miR-466 mimic, transfection of miR-466 mimic remarkably downregulated mRNA and protein levels of CXCR4 (Fig. 4D). To confirm that CXCR4 regulates human glioma cell proliferation and apoptosis, we transfected pCDNA-CXCR4, shCXCR4, or a negative control into glioma cells and analyzed the cells using MTT, BrdU proliferation, flow cytometry assay, and cell cycle assay. Distinctly higher cell viability and proliferation, as well as clearly reduced apoptosis, and G1/S cell cycle transition were observed in the shCXCR4 group relative to the pcDNA-CXCR4 or control groups (Fig. 4E-H).

\section{RMRP silencing or miR-466 mimic inhibits tumor growth in vivo}

In order to determine the therapeutic potential of shRMRP or miR-466 mimic for the treatment of human glioma, nude mice were subcutaneously injected with U373MG cells that were successfully transfected with corresponding shRNA or miRNA mimic. Our data showed that the tumors were isolated from nude mice and measured grew more slowly in the shRMRP and miR-466 mimic group as compared to the control group (Fig. 5A-C). Notably, the tumor volume and size of mice injected with the miR-466 mimic transfected cells were smaller as compared to that of mice injected with the shRMRP-transfected cells (Fig. 5A-C). This result confirmed that RMRP could be potential for the therapeutic target for human glioma in vivo.

\section{Discussion}


The dysregulated expression of IncRNA may provide a favorable landscape for cell growth by affecting epigenetic information, leading to the uncontrolled growth of tumor cells, and thus to the occurrence and development of tumors. Moreover, effective control of cell survival and cell proliferation is the key to the prevention and treatment of cancer [18]. Therefore, identifying cancer-related IncRNAs and studying their clinical significance and molecular regulatory mechanisms may be able to fill a missing piece in the wellknown mystery of carcinogenesis and tumor inhibition network. The RNA component of mitochondrial RNA processing endoribonuclease (RMRP), a IncRNA, was first discovered in cartilage-hair hypoplasia $(\mathrm{CHH})$, an autosomal recessive inherited disease [19]. RMRP is primarily expressed in a variety of human tissues, and existed in the nucleus, nucleolus and mitochondria, which contributes to early stages of embryonic development $[20,21]$. Recent study have found that RMRP silencing can significantly inhibit the proliferation and differentiation of gastric cancer cells in vitro and in vivo, indicating that it plays an important role in the occurrence and development of gastric cancer, and has potential as a biomarker and drug target for the diagnosis and treatment of gastric cancer [22]. Therefore, our study systematically investigated the regulatory networks and molecular mechanisms of RMRP in the development and progression of human glioma.

Subsequently, our data revealed that IncRNA RMRP promoted cell proliferation but reduced apoptosis in human glioma cells via targeting miR-466. Amounting evidence indicated that miR-466 was associated with tumor progression in various cancers. For example, $\mathrm{Li}$ and colleagues reported that miR-466 was downregulated in tumor tissues in prostate carcinoma patients, and overexpression of miR-466 resulted in decreased rates of cell migration and invasion [23]. Another study in hepatocellular carcinoma revealed that miR-466 was obviously decreased in tissues and cell lines, and restoration of miR-466 expression could inhibit the cell proliferation but induced apoptosis in vitro, as well as impeded xenograft tumor growth in vivo [13]. Consistent with these studies, our data also demonstrated that miR-466 was significantly decreased in glioma tissues and cells, while miR-466 overexpression suppressed cell viability, proliferation even tumor growth whereas promoted apoptotic rate in experiments in vitro and in vivo. These results confirmed that RMRP was negatively interacted with miR-466, contributed to the promotion effect on glioma progression.

In addition, several IncRNA have been described as regulators of cell proliferation and cell cycle. Most of the studies are related to IncRNA-induced cell cycle arrest, and these IncRNA have been confirmed to target multiple components of the cell cycle, including cyclin D1, cyclin E, CDK4, CDK2, and E2F3. However, the molecular mechanisms of IncRNA promoting cell cycle and eliciting G1/S transition have not been reported, and the biological relevance of such a IncRNA-mediated regulation of the cell cycle machinery in vivo remains unclear. CDK4 and CDK6 are activated by D-type cyclin complex formation that acts as a cell cycle growth sensor, whereas CDK2 binds to cyclin E or cyclin A, and also regulates G1$S$ phase [24]. In this study, our results found that IncRNA RMRP could induce G1/S cell cycle transition through cyclin D-CDK4/6 complex upregulation but not cyclin E-CDK2 complexes. Notably, most previous studies have observed that CDK4/6 and CDK2 are upregulated and downregulated respectively and simultaneously in the cell cycle $[25,26]$. However, RMRP-promoted cell cycle only upregulated CDK4 
expression, whereas CDK2 expression was unchanged. This may indicate that RMRP promotes cell proliferation via targeting CDK4, but the detailed mechanisms need to be further elucidated.

Further experiments showed that CXCR4 was a candidate of miR-46a. Previous studies manifested that the increased expression of CXCR4 was confirmed in many tumors. For example, CXCR4 has been reported that as an oncogene fulfill the function in cell proliferation, migration, as well as metastasis in breast cancer [27]. Also, the meta-analysis demonstrated that CXCR4 over-expression was highly correlated with poor survival in various types of cancers, including esophageal cancer, renal cancer, gynecologic cancer, and liver cancer [28]. Consistent with the previous documented, our data also revealed that CXCR4 was apparently increased in human glioma cell lines. Furthermore, the functional experiments indicated that CXCR4 overexpression partly counteracted the promotion impacts on cell proliferation as well as facilitate to G1/S cell cycle transition, but reduce the apoptosis, indicating the promoting role of CCT6A in glioma progression. These data implied that RMRP promoted human glioma progression via sponging miR-466 and positively regulating CXCR4 expression. However, the deficiency of our study is that we only evaluated RMRP and miR-466 as therapeutic targets for treating glioma in vivo, without the evaluation of CXCR4, since CXCR4 has been widely reported as a cancer therapeutic target $[29,30]$. In future experimental design, we will design more and more detailed experiments to comprehensively evaluate the treatment potential of these targets for human glioma.

In conclusion, our study provides a new insight on the regulatory mechanisms of IncRNA RMRP on human glioma progression. Our data were first to highlight high RMRP expression levels in human glioma and demonstrate that RMRP promotes cell proliferation, G1/S cell cycle transition but inhibits apoptosis in human glioma by targeting miR-466/CXCR4 axis. Overall, our data revealed that RMRP and miR-466 may be the potential targets of human glioma treatment in the future.

\section{Declarations}

\section{Compliance with Ethical Standards}

\section{Funding}

Not applicable

\section{Conflict of interest}

The authors have completed the ICMJE uniform disclosure form, and confirmed that there is no conflict of interests.

\section{Ethics approval}

This study was approved by the Institutional Research Ethics Committee of the First Affiliated of Chengdu Medical College (IRB number: 20140332) 


\section{Informed consent}

All participants provided written informed consent.

\section{Author Contributions}

Jie Zhang conceived and designed the experiments; Jie Zhang, Ling He, Xun Xia, Lie Zhang and Enren Wang performed the experiments and analyzed the data; Jie Zhang and Ling He wrote the manuscript.

\section{Data availability statement}

The authors confirm that all data underlying the findings are fully available without restriction. All relevant data are within the paper files.

\section{Abbreviations}

Long non-coding RNA, IncRNA; RNA component of mitochondrial RNA processing endoribonuclease, RMRP; cartilage-hair hypoplasia, $\mathrm{CHH}$; microRNA-466, miR-466; normal human astrocytes, NHAs; Noncoding RNAs, ncRNAs.

\section{Consent for publication}

Not applicable.

\section{Acknowledgements}

Not applicable.

\section{References}

1. Bi J, Chowdhry S, Wu S, Zhang W, Masui K, Mischel PS. Altered cellular metabolism in gliomas - an emerging landscape of actionable co-dependency targets. Nature reviews Cancer. 2020;20(1):57-70. doi:10.1038/s41568-019-0226-5. PubMed PMID: 31806884. Epub 2019/12/07.

2. Peng WX, Koirala P, Mo YY. LncRNA-mediated regulation of cell signaling in cancer. Oncogene. 2017;36(41):5661-7. doi:10.1038/onc.2017.184. PubMed PMID: 28604750; PubMed Central PMCID: PMCPMC6450570. Epub 2017/06/13.

3. Bhan A, Soleimani M, Mandal SS. Long Noncoding RNA and Cancer: A New Paradigm. Cancer research. 2017;77(15):3965-81. doi:10.1158/0008-5472.can-16-2634. PubMed PMID: 28701486. Epub 2017/07/14.

4. Liu ZZ, Tian YF, Wu H, Ouyang SY, Kuang WL. LncRNA H19 promotes glioma angiogenesis through miR-138/HIF-1a/VEGF axis. Neoplasma. 2020;67(1):111-8. Epub 2019/11/30. doi: 10.4149/neo_2019_190121N61. PubMed PMID: 31777264. 
5. Fu C, Li D, Zhang X, Liu N, Chi G, Jin X. LncRNA PVT1 Facilitates Tumorigenesis and Progression of Glioma via Regulation of MiR-128-3p/GREM1 Axis and BMP Signaling Pathway. Neurotherapeutics: the journal of the American Society for Experimental NeuroTherapeutics. 2018;15(4):1139-57. Epub 2018/08/19. doi:10.1007/s13311-018-0649-9. PubMed PMID: 30120709; PubMed Central PMCID: PMCPMC6277294.

6. Yang M, Ke H, Zhou W. LncRNA RMRP Promotes Cell Proliferation and Invasion Through miR613/NFAT5 Axis in Non-Small Cell Lung Cancer. OncoTargets therapy. 2020;13:8941-50. doi:10.2147/ott.s255126. PubMed PMID: 32982286; PubMed Central PMCID: PMCPMC7494237. Epub 2020/09/29.

7. Cao HL, Liu ZJ, Huang PL, Yue YL, Xi JN. IncRNA-RMRP promotes proliferation, migration and invasion of bladder cancer via miR-206. Eur Rev Med Pharmacol Sci. 2019;23(3):1012-21. Epub 2019/02/20. doi: 10.26355/eurrev_201902_16988. PubMed PMID: 30779067.

8. Hongfeng Z, Andong J, Liwen S, Mingping B, Xiaowei Y, Mingyong L, et al. IncRNA RMRP knockdown suppress hepatocellular carcinoma biological activities via regulation miRNA-206/TACR1. Journal of cellular biochemistry. 2020;121(2):1690-702. doi:10.1002/jcb.29404. PubMed PMID: 31579977. Epub 2019/10/04.

9. Rolle K. miRNA Multiplayers in glioma. From bench to bedside. Acta biochimica Polonica. 2015;62(3):353 - 65. Epub 2015/08/27. doi: 10.18388/abp.2015_1072. PubMed PMID: 26307768.

10. Zhou Q, Liu J, Quan J, Liu W, Tan H, Li W. MicroRNAs as potential biomarkers for the diagnosis of glioma: A systematic review and meta-analysis. Cancer Sci. 2018;109(9):2651-9. doi:10.1111/cas.13714. PubMed PMID: 29949235; PubMed Central PMCID: PMCPMC6125451. Epub 2018/06/28.

11. Paraskevopoulou MD, Hatzigeorgiou AG. Analyzing MiRNA-LncRNA Interactions. Methods in molecular biology (Clifton, NJ). 2016;1402:271 - 86. Epub 2016/01/02. doi: 10.1007/978-1-49393378-5_21. PubMed PMID: 26721498.

12. Tong F, Ying Y, Pan H, Zhao W, Li H, Zhan X. MicroRNA-466 (miR-466) functions as a tumor suppressor and prognostic factor in colorectal cancer (CRC). Bosnian journal of basic medical sciences. 2018;18(3):252-9. doi:10.17305/bjbms.2018.2376. PubMed PMID: 29338680; PubMed Central PMCID: PMCPMC6087559. Epub 2018/01/18.

13. Jia C, Tang D, Sun C, Yao L, Li F, Hu Y, et al. MicroRNA-466 inhibits the aggressive behaviors of hepatocellular carcinoma by directly targeting metadherin. Oncol Rep. 2018;40(6):3890-8. doi:10.3892/or.2018.6763. PubMed PMID: 30542714. Epub 2018/12/14.

14. Cao W, Fang L, Teng S, Chen H, Liu T. MicroRNA-466 inhibits osteosarcoma cell proliferation and induces apoptosis by targeting CCND1. Experimental and therapeutic medicine. 2018;16(6):511722. Epub 2018/12/14. doi: 10.3892/etm.2018.6888. PubMed PMID: 30546411; PubMed Central PMCID: PMCPMC6256845.

15. Louis DN, Ohgaki H, Wiestler OD, Cavenee WK, Burger PC, Jouvet A, et al. The 2007 WHO classification of tumours of the central nervous system. Acta Neuropathol. 2007;114(2):97-109. 
doi:10.1007/s00401-007-0243-4. PubMed PMID: 17618441; PubMed Central PMCID: PMCPMC1929165. Epub 2007/07/10.

16. Asghar U, Witkiewicz AK, Turner NC, Knudsen ES. The history and future of targeting cyclindependent kinases in cancer therapy. Nature reviews Drug discovery. 2015;14(2):130-46. doi:10.1038/nrd4504. PubMed PMID: 25633797; PubMed Central PMCID: PMCPMC4480421. Epub 2015/01/31.

17. Sherr CJ, Beach D, Shapiro GI. Targeting CDK4 and CDK6: From Discovery to Therapy. Cancer discovery. 2016;6(4):353-67. doi:10.1158/2159-8290.cd-15-0894. PubMed PMID: 26658964; PubMed Central PMCID: PMCPMC4821753. Epub 2015/12/15.

18. Shi X, Sun M, Liu H, Yao Y, Song Y. Long non-coding RNAs: a new frontier in the study of human diseases. Cancer letters. 2013;339(2):159-66. doi:10.1016/j.canlet.2013.06.013. PubMed PMID: 23791884. Epub 2013/06/25.

19. Huang W, Thomas B, Flynn RA, Gavzy SJ, Wu L, Kim SV, et al. DDX5 and its associated IncRNA Rmrp modulate TH17 cell effector functions. Nature. 2015;528(7583):517-22. doi:10.1038/nature16193. PubMed PMID: 26675721; PubMed Central PMCID: PMCPMC4762670. Epub 2015/12/18.

20. Rosenbluh J, Nijhawan D, Chen Z, Wong KK, Masutomi K, Hahn WC. RMRP is a non-coding RNA essential for early murine development. PloS one. 2011;6(10):e26270.

doi:10.1371/journal.pone.0026270. PubMed PMID: 22039455; PubMed Central PMCID: PMCPMC3198473. Epub 2011/11/01.

21. Chang DD, Clayton DA. A novel endoribonuclease cleaves at a priming site of mouse mitochondrial DNA replication. EMBO J. 1987;6(2):409-17. Epub 1987/02/01. PubMed PMID: 3582365; PubMed Central PMCID: PMCPMC553411.

22. Shao Y, Ye M, Li Q, Sun W, Ye G, Zhang X, et al. LncRNA-RMRP promotes carcinogenesis by acting as a miR-206 sponge and is used as a novel biomarker for gastric cancer. Oncotarget. 2016;7(25):37812-24. doi:10.18632/oncotarget.9336. PubMed PMID: 27192121; PubMed Central PMCID: PMCPMC5122351. Epub 2016/10/23.

23. Li G, Zhang Y, Mao J, Hu P, Chen Q, Ding W, et al. LncRNA TUC338 is overexpressed in prostate carcinoma and downregulates miR-466. Gene. 2019;707:224 - 30. Epub 2019/05/16. doi: 10.1016/j.gene.2019.05.026. PubMed PMID: 31085276.

24. Matsumoto M, Furihata M, Ishikawa T, Ohtsuki Y, Ogoshi S. Comparison of deregulated expression of cyclin D1 and cyclin E with that of cyclin-dependent kinase 4 (CDK4) and CDK2 in human oesophageal squamous cell carcinoma. British Journal of Cancer. 80(1):256-61.

25. Sarcevic B. Estrogen-induced Activation of Cdk4 and Cdk2 during G1-S Phase Progression Is Accompanied by Increased Cyclin D1 Expression and Decreased Cyclin-dependent Kinase Inhibitor Association with Cyclin E-Cdk2. Journal of Biological Chemistry. 272(16):10882-94.

26. Wierã,D L, Rosseland CM, Lindeman B, Oksvold MP, Grã,Svik H, Skarpen E, et al. CDK2 regulation through PI3K and CDK4 is necessary for cell cycle progression of primary rat hepatocytes. Cell Prolif. 2010;40(4):475-87. 
27. Xu C, Zhao H, Chen H, Yao Q. CXCR4 in breast cancer: oncogenic role and therapeutic targeting. Drug design, development and therapy. 2015;9:4953-64. Epub 2015/09/12. doi: 10.2147/dddt.s84932. PubMed PMID: 26356032; PubMed Central PMCID: PMCPMC4560524.

28. Zhao H, Guo L, Zhao H, Zhao J, Weng H, Zhao B. CXCR4 over-expression and survival in cancer: a system review and meta-analysis. Oncotarget. 2015;6(7):5022-40. doi:10.18632/oncotarget.3217. PubMed PMID: 25669980; PubMed Central PMCID: PMCPMC4467131. Epub 2015/02/12.

29. Eckert F, Schilbach K, Klumpp L, Bardoscia L, Sezgin EC, Schwab M, et al. Potential Role of CXCR4 Targeting in the Context of Radiotherapy and Immunotherapy of Cancer. Frontiers in immunology. 2018;9:3018. doi:10.3389/fimmu.2018.03018. PubMed PMID: 30622535; PubMed Central PMCID: PMCPMC6308162. Epub 2019/01/10.

30. Lecavalier-Barsoum M, Chaudary N, Han K, Koritzinsky M, Hill R, Milosevic M. Targeting the CXCL12/CXCR4 pathway and myeloid cells to improve radiation treatment of locally advanced cervical cancer. International journal of cancer. 2018;143(5):1017-28. doi:10.1002/ijc.31297. PubMed PMID: 29417588. Epub 2018/02/09.

\section{Figures}
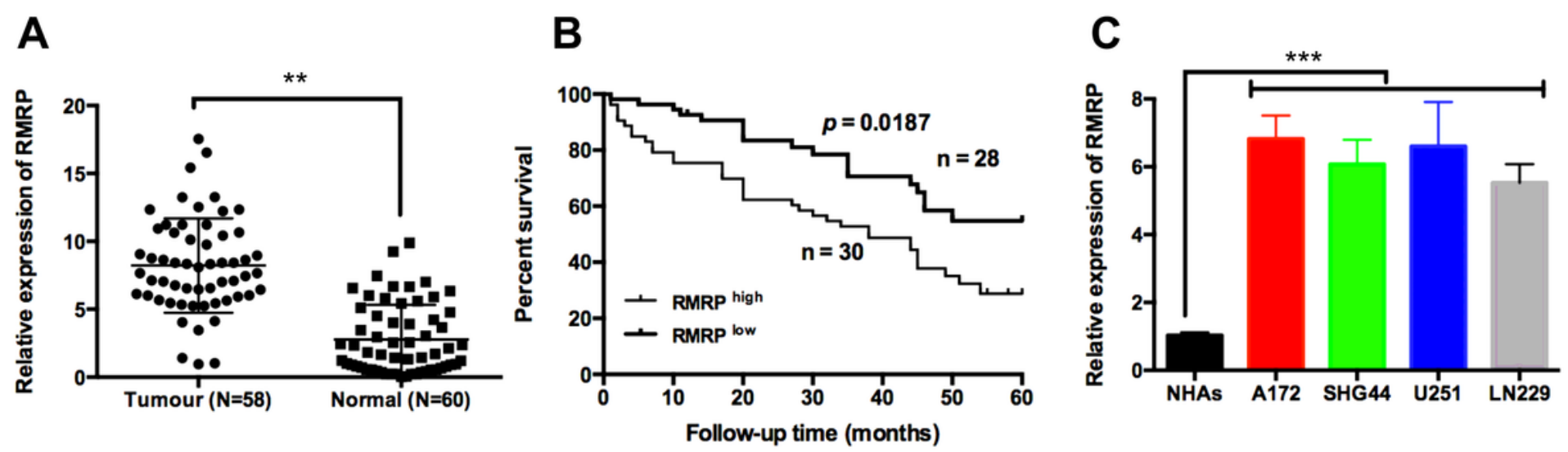

\section{Figure 1}

The increased expression levels of IncRNA RMRP is determined in human glioma tissues. The expression levels of RMRP in 58 pairs of human glioma tissues and their adjacent, non-cancerous normal tissues (Normal) (A) and the human glioma cell lines A172, SHG44, U251, LN229 and the normal human astrocytes (NHAs) (C) are detected by qRT-PCR. U6 is used as the internal control. (C) Overall survival based on RMRP expression levels in glioma patients was represented by Kaplan-Meier survival analysis. All results were presented as the mean $\pm \mathrm{SD}$ and experiments were conducted in triplicate. ${ }^{*} \mathrm{P}<0.01$ and $\star \star * P<0.001$. 

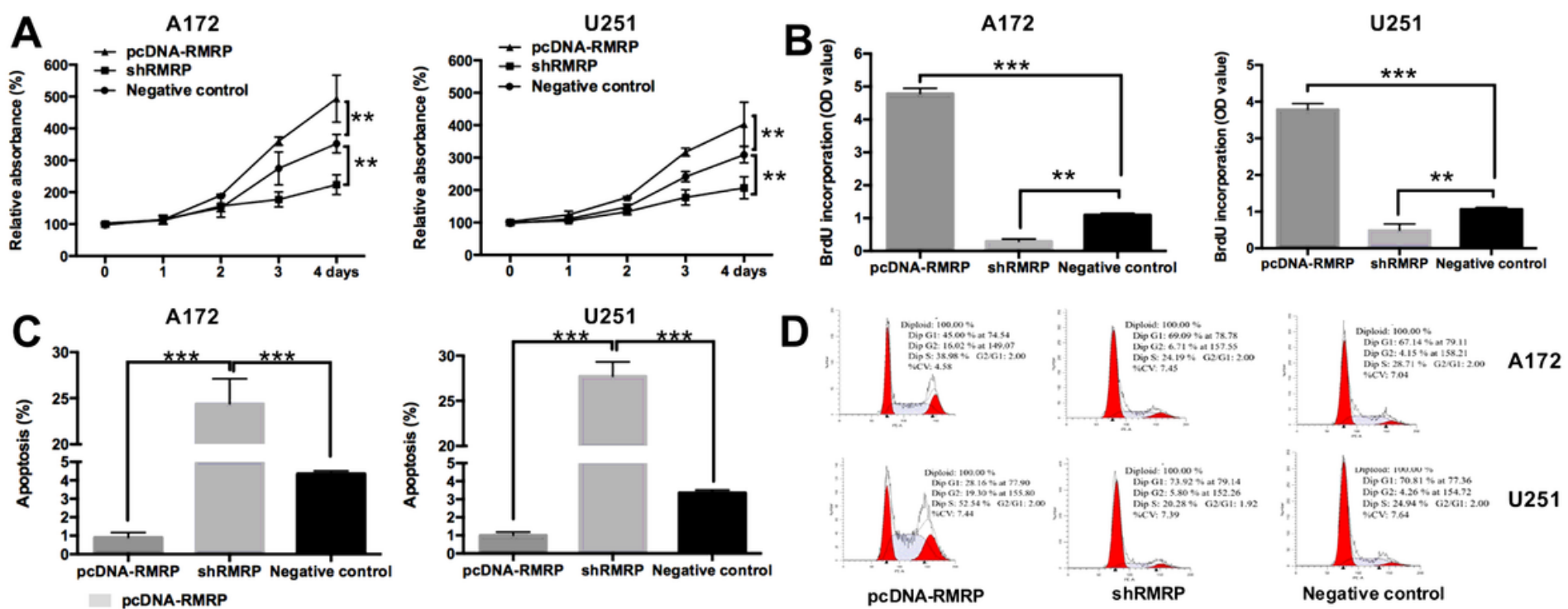

E $=\begin{aligned} & \text { shRMRP } \\ & \text { Negative control }\end{aligned}$
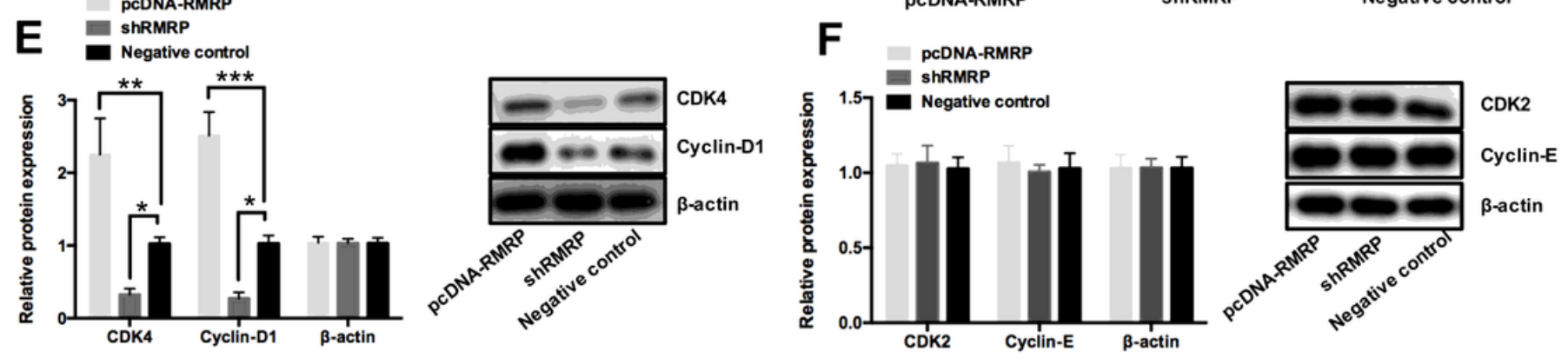

Figure 2

IncRNA RMRP promotes proliferation, G1/S cell cycle transition and inhibits apoptosis in human glioma cells. (A) pcDNA-RMRP is transfected into A172 and U251 cells and induces an obviously higher proliferation rate than shRMRP transfected group and negative control group. (B) The significantly increased cell proliferation levels in A172 and U251 cells transfected with pcDNA-RMRP plasmid and the obviously down-regulated cell proliferation of shRMRP transfected group compared with that in the controls were observed by ELISA-BrdU assay. (C) pcDNA-RMRP or shRMRP is transfected into A172 and U251 cells and shRMRP triggers a significantly high apoptosis compared with controls. (D) The effects of pcDNA-RMRP or shRMRP transfection on cell cycle in A172 and U251 cells using flow cytometry. (E) and (F) The cyclin D-CDK4/6 and cyclin E-CDK2 complex protein expressions were determined by Western blotting using $\beta$-actin as an internal control. ${ }^{*} P<0.05$, ${ }^{\star} \mathrm{P}<0.01$, and ${ }^{\star \star *} \mathrm{P}<0.001$. 
A

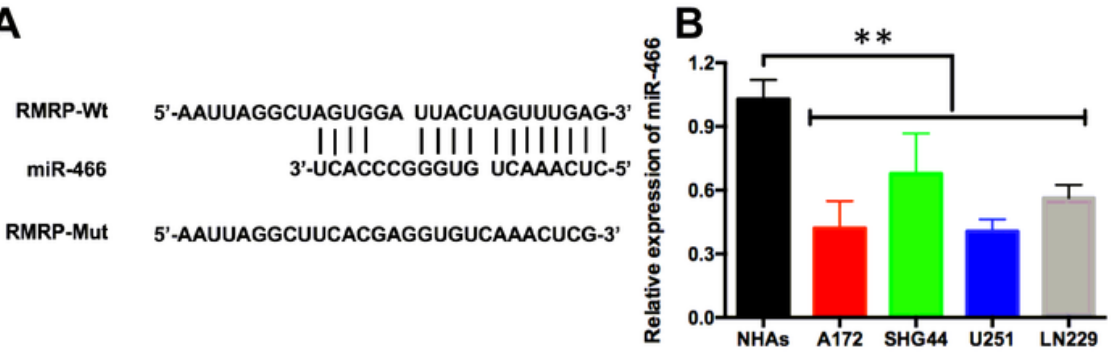

D
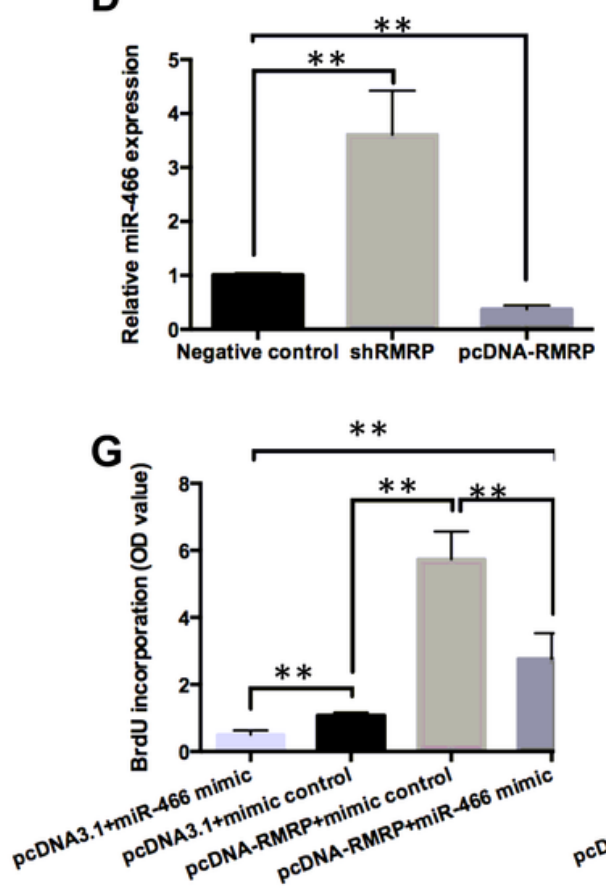

E

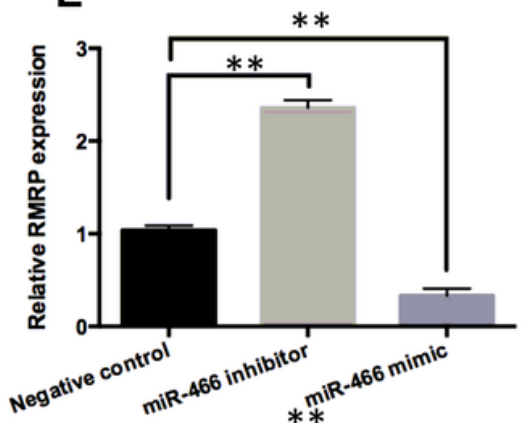

$$
\text { H }
$$

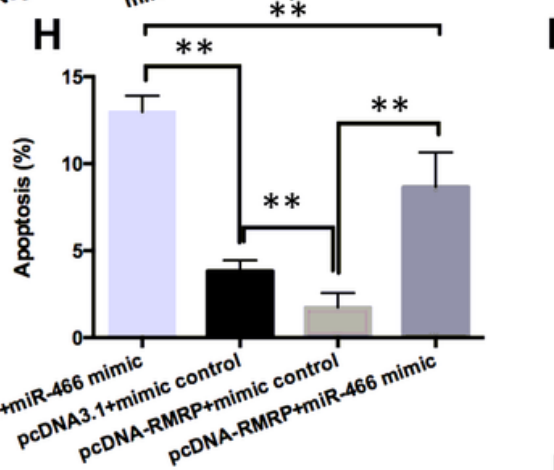

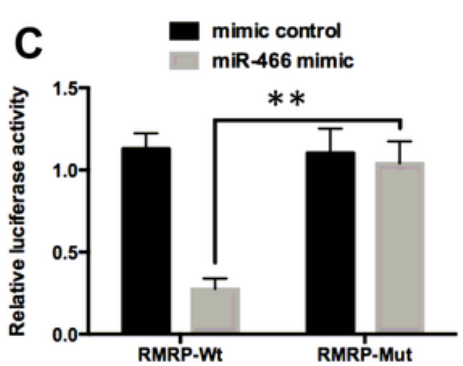

$F=$ pcDNA-RMRP+miR 466 mimic

- pcDNA-RMRP+mimic control

$\rightarrow$ pcDNA3.1+mimic control

$\rightarrow$ pcDNA3.1+miR-466 mimic
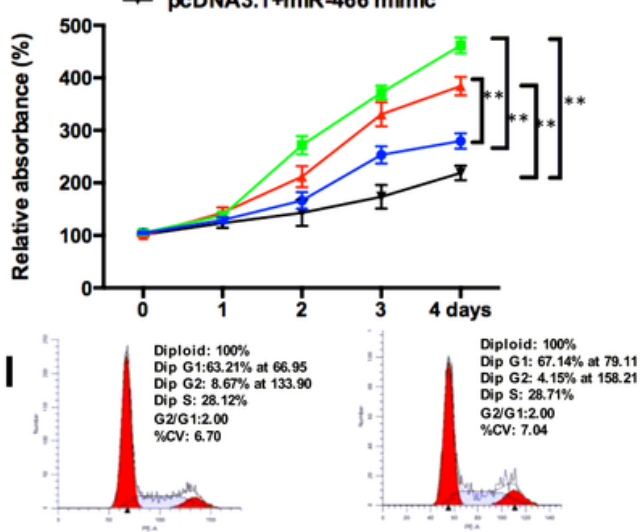

pcDNA3.1+miR-466 mimic pcDNA3.1+mimic control

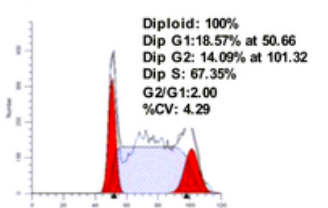

pcDNA-RMRP+mimic control pcDNA-RMRP+miR-466 mimic

\section{Figure 3}

miR-466 is a direct target of RMRP. (A) Bioinformatics-based target prediction analysis shows that a potential target of RMRP is called as miR-466. (B) The expression levels of miR-466 are markedly decreased in human glioma cell lines compared with those in NHAs. (C) Luciferase reporter assay shows that in the miR-466 mimic group, the significantly decreased luciferase activity driven by RMRP is observed compared with that in the negative control and RMRP-MUT group. (D) The expression levels of miR-466 were measured in A172 cells transfected with the pcDNA-RMRP or shRMRP. (E) The expression levels of RMRP were measured in A172 cells transfected with the miR-466 mimic or miR-466 inhibitor. MiR-466 reverses the biological function of RMRP on human glioma cell. (F) Cell MTT assay, (G) cell proliferation assay, $(\mathrm{H})$ apoptosis assay and $(\mathrm{I})$ cell cycle assay were performed in human glioma cells transfected with pcDNA-RMRP + miR-466 mimics, pcDNA-RMRP + mimic control, pcDNA3.1 + miR-466 mimics and pcDNA3.1 + mimic control. All results were presented as the mean \pm SD and experiments were conducted in triplicate, ${ }^{\star *} p<0.01$ and $* \star * p<0.001$. 
A

CXCR4 3'UTR-WT 5'-AGUUGAAAAAAGGUUGGUUUGAG... miR-466 IIIIIII

CXCR4 3'UTR-MUT 5'- AGUUGAAAAAUCGUUGUCACCGU...
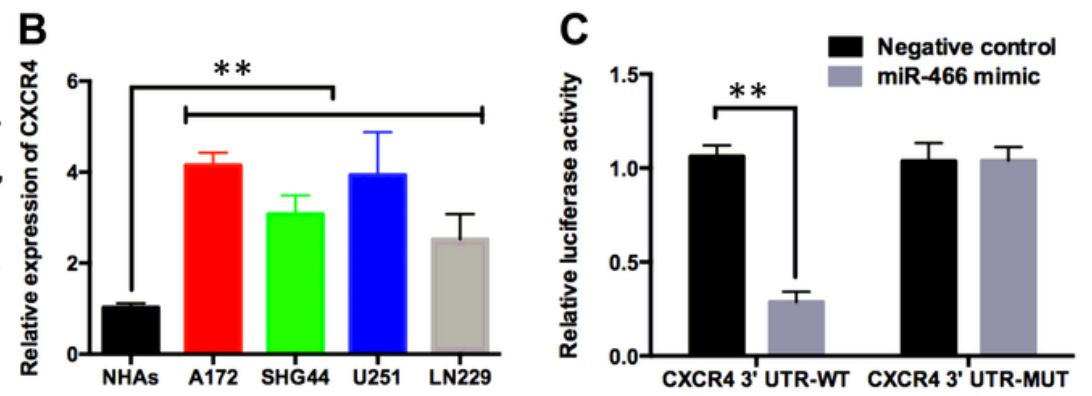
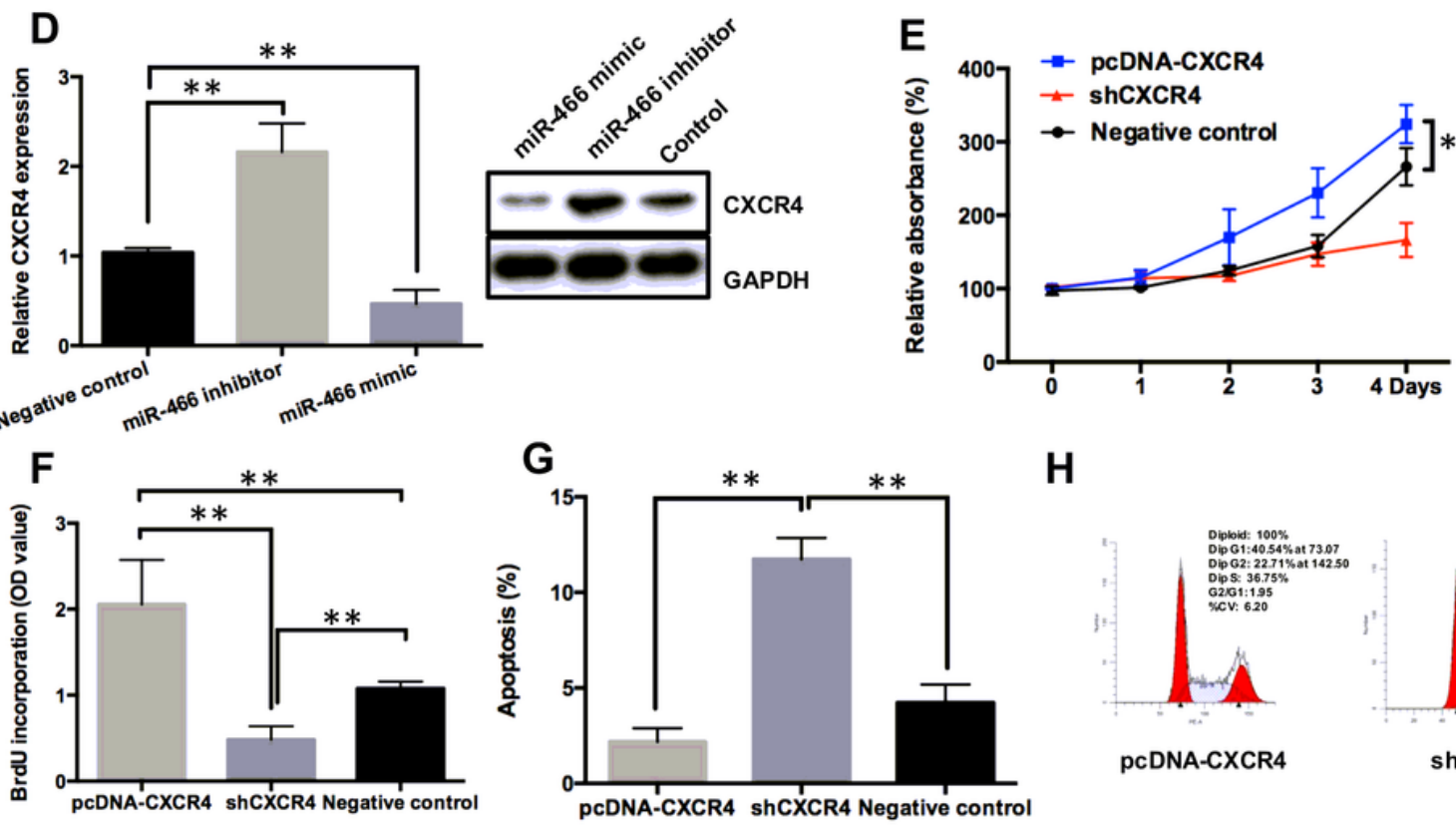

H
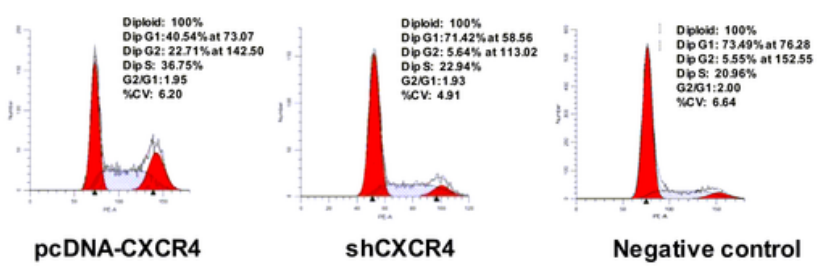

Figure 4

CXCR4 is a direct target of miR-466. (A) Bioinformatics-based target prediction analysis shows that a potential target of miR-466 is called as CXCR4. (B) The expression levels of CXCR4 are markedly increased in human glioma cell lines compared with those in NHAs. (C) Luciferase reporter assay shows that in the miR-466 mimic group, the significantly decreased luciferase activity driven by CXCR4 is observed compared with that in the negative control and CXCR4-MUT group. (D) The expression levels of CXCR4 were measured in A172 cells transfected with the miR-466 mimic or miR-466 inhibitor. CXCR4 expression promotes cell proliferation and decreases apoptosis on human glioma cells. (E) An obviously higher cell viability rate is observed in the pcDNA-CXCR4 group than that transfected with shCXCR4 as control. (F) A significantly higher cell proliferation is observed in the pCDNA-CXCR4 group than control. (G) The significantly down-regulated apoptosis rate is detected in the pcDNA-CXCR4 group compared with control. $(\mathrm{H})$ Cell cycle of various groups was detected using flow cytometry. Expression of CXCR4 could block cell cycle in $S$ phase. ${ }^{* *} p<0.01$. 


\section{A}

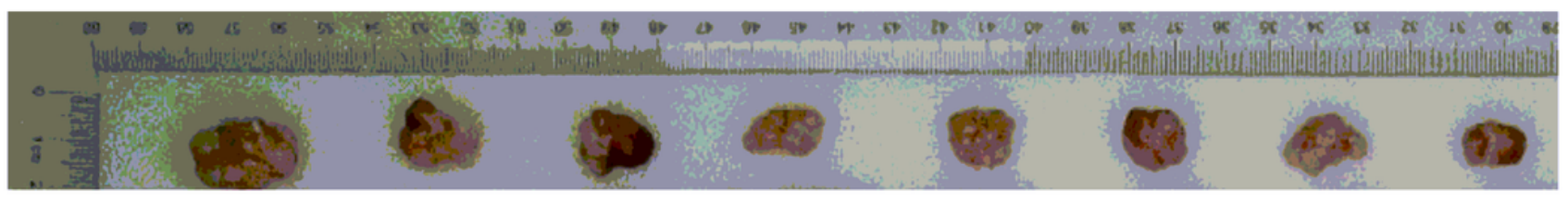

shctrl

mimic control

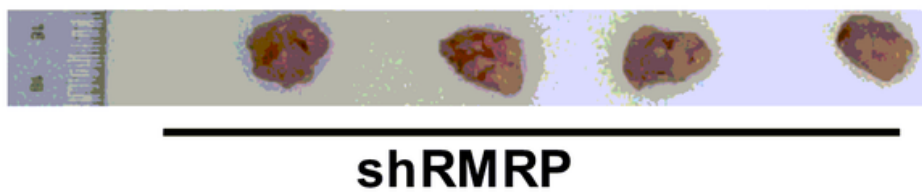

B
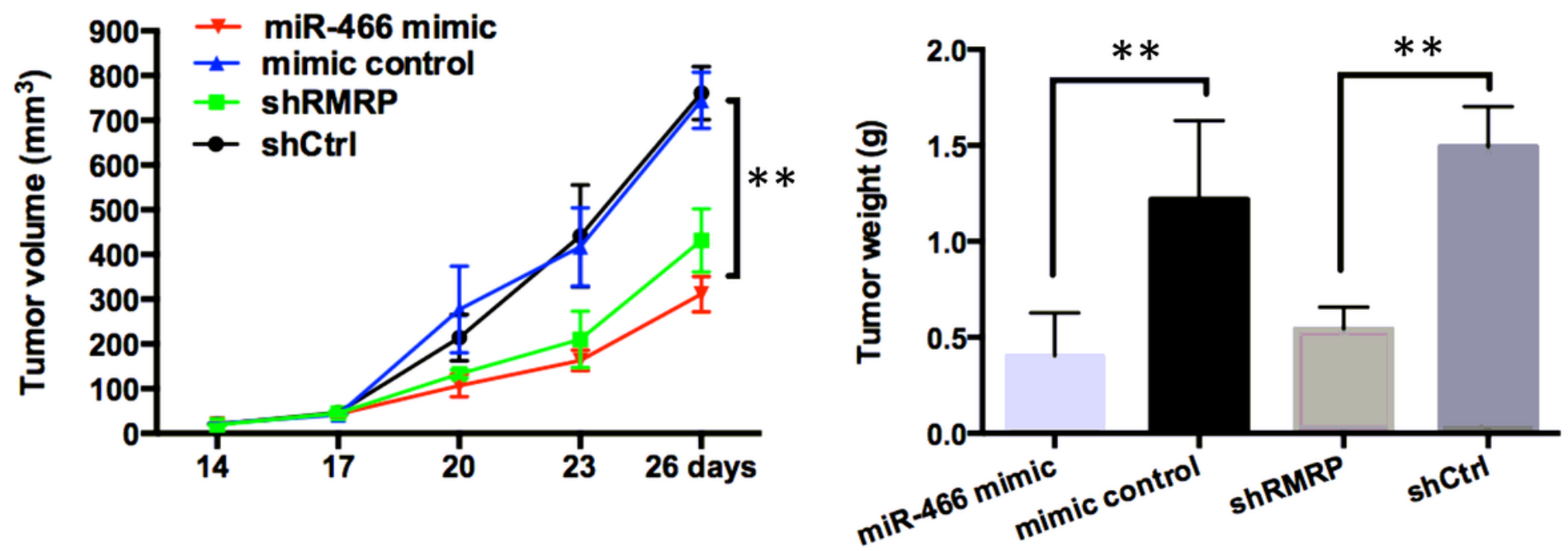

Figure 5

RMRP knockdown and miR-466 expression suppresses the growth of human glioma cells in vivo. (A) Tumor volume after injection of U373MG cells transfected with shRMRP, shCtrl, miR-466 or mimic control.

(B) After 2 weeks, the tumor volumes were calculated after 5 tests by measure the tumor sizes (day 14,17 , 20, 23 and day 26. (C) The average weight of the tumor at 5 weeks after cell injection. ${ }^{\star *} \mathrm{P}<0.05$. 